\title{
Direct RF Sampling GNSS Receiver Design and Jitter Analysis
}

\author{
Guillaume Lamontagne $^{1}$, René Jr. Landry ${ }^{2}$, Ammar B. Kouki ${ }^{2}$ \\ ${ }^{1}$ MDA Corporation, Satellite Systems Division, Sainte-Anne-de-Bellevue, Canada; ${ }^{2}$ École de technologie supérieure, LACIME \\ Laboratory, Montréal, Canada. \\ Email: guillaume.lamontagne@mdacorporation.com, ReneJr.Landry@etsmtl.ca,ammar.kouki@etsmtl.ca
}

Received August 25 $5^{\text {th }}, 2012$; revised September $30^{\text {th }}, 2012$; accepted October $17^{\text {th }} 2012$

\begin{abstract}
This paper describes the design of a flexible Direct RF Sampling based GNSS receiver as well as its use for the verification of jitter effects on various performance metrics. The proposed architecture allows the sampling and the real-time digital signal processing of real GNSS signals. The analysis of the measurements obtained from this system validates theoretical formulations from which the sampling jitter limit is established in order not to impact the GNSS signal's detection.
\end{abstract}

Keywords: Direct RF Sampling; GNSS Receiver; Jitter

\section{Introduction}

Over the last decades, numerous governments invested a great deal of efforts and funds in designing, launching and operating complex Global Navigation Satellite Systems (GNSS). Since the first experimental Global Positioning Satellite (GPS) satellite was launched in 1978 by the United States of America (USA), many countries decided to join the race and are currently trying to gain independent access to global satellite navigation by launching their own satellite constellation system. Russia was the second country to design such a system, called GLONASS, and recently enhanced its constellation with its latest launch at the end of 2011 [1]. The European Union is also developing its own constellation and currently has two satellites deployed in orbit and they are going through their commissioning phase [2]. The latest to join the race is the Compass system, designed by China, which is currently ahead of the Galileo program, with eleven spacecrafts launched as of April 2012 [3].

Basically, the navigation signals transmitted by those systems follow the same trilateration positioning principles as the GPS but they differ in terms of signal definition (frequency, bandwidth and modulation). These differences result in GPS receivers' design (RF front-end and digital signal processing) not being fully compatible with the other systems. On the other hand, users are looking for higher precision, better satellite visibility, greater integrity and more robustness. The combination of the various GNSS signals in a single GNSS receiver can help satisfy the users' needs to some extent. There- fore, the logical step is to design a universal GNSS receiver that could receive and process the signals from various satellite navigation systems. However, one limitation in the design of a universal GNSS receiver is the RF front-end, which may be complex and/or technically limited for covering all potential GNSS bands and signals. Furthermore, the sensitivity of the RF front-end is essential in several applications using high sensitivity receivers.

The objective of this paper is twofold. First, a prototype using Direct RF Sampling (DRFS) is proposed as a first step towards a universal GNSS receiver. A wideband RF front-end first amplifies and filters the GPS signals, which are later sampled by a high-speed analog-to-digital converter. The signals are then down-converted and filtered further in the digital domain and then sent to a custom GPS receiver. This GNSS receiver, to be referred to as the RxGNSS hereafter, performs the data demodulation required to find the Position, Velocity and Time (PVT) navigation solution. It was designed and built during previous studies at the LACIME laboratory of ÉTS as described in [4].

The first published work on DRFS that applied to the GPS dates back to 1994 [5] but the results were limited by the available sampling and processing technologies. Since then, many authors developed the concept, such as [6-9], proposing DRFS prototypes for GNSS signals along with detailed studies using post-processed data. In [6], Thor and Akos describe a DRFS prototype to capture both the GPS L1 C/A and the GPS L3 nuclear detection 
signal at $1381.05 \mathrm{MHz}$. The sampling frequency used is 73.45 MHz and an FPGA processes the data and transfers it to a PC for post-processing. The prototype described in [9], similar to the one proposed in this paper, is one of the most advanced since it captures both the GPS L1 C/A, L1 P(Y) and L2 P(Y) signals. The prototype samples the GPS signals at a frequency of up to 99.23 $\mathrm{MHz}$ and stores the data on a PC in order to proceed with acquisition and tracking using a Matlab ${ }^{\mathrm{TM}}$ based software receiver.

The Direct RF sampling prototype presented herein represent a significant step forward compared to previous work as it is the first one reported to have the capability to process the data in real-time. This is accomplished both in the RF front-end using an FPGA and in the RxGNSS software receiver as described in Section 3. Furthermore, the sampling frequency used for the proposed prototype is tripled when compared to that of the one of [9], for instance. Even though this increase is not necessarily required for the GPS L1 C/A signal, it demonstrates the feasibility of such a high sampling frequency in the context of a real-time DRFS receiver. Both improvements are of high interest for multiband GNSS receivers as well as several mass market applications, including personal mobiles devices, which usually capture multiple services at different frequencies (UMTS, WIFI, Bluetooth, GPS, etc.).

The details of the Direct RF Sampling technique are presented in Section 2 and the practical implementation is detailed in Sections 4 and 5 for the RF and the digital architectures respectively. The prototype description provides a better understanding of the design drivers to focus on in order to limit as much as possible jitter influence on the GNSS signals.

Second, the specific effects of the sampling jitter are studied for the designed prototype. The question of the jitter limits for a DRFS receiver for GNSS is well described in many past publications, including [10-12]. In the latter, Dempster and Bilal define a maximum allowable jitter for a BPSK signal sampled using a DRFS architecture. The theoretical analysis of [12], from which a mathematical model is derived in Section 3 for the proposed prototype, is a fruitful contribution providing guidelines to design a DRFS architecture. In the model of [12], the limit itself is selected based on an educated guess about the effect of the jitter compared to the effect of thermal noise on the receiver's positioning accuracy. However, the paper, along with other publications on the subject, does not present experimental data to validate the analysis. An interesting experimentation is presented in [7], in which the jitter analysis of [12] is summarized and the resulting guideline is used to design a DRFS proto-type to capture the GPS L1 C/A signal. The performance of this prototype is compared to a classical super-heterodyne front-end using navigation data bits inphase and in-quadrature samples. Two different sampling clocks are used to assess the jitter effect but the actual sampling jitter limit is not experimentally studied.

The DRFS prototype proposed in this paper allows, for the first time, the capture of experimental data with a variable sampling jitter below, around and above the theoretical limit set by [12]. Indeed, in Section 6, the jitter effects are evaluated using real time GPS signals in order to demonstrate the validity of the limit, taking into account the specificities of the DRFS prototype as described in Section 3. This validity was previously accepted based on the theoretical studies only but the data gathered by the proposed prototype brings a concrete demonstration that the limit is sufficient for Direct RF Sampling GNSS receivers.

\section{The Direct RF Sampling Technique}

\subsection{GNSS Receivers Architecture}

The commonly used approach for a GNSS receiver RF front-end is to employ a super-heterodyne architecture which down-converts each one of the satellite's signals bands with a different Local Oscillator (LO) prior to sampling, thus requiring one RF front-end per GNSS signal's band. Figure 1 shows this super-heterodyne architecture for the reception of two GNSS signals in two distinct frequency bands.

As the number of signals grows when new GNSS signals become operational, this traditional approach rapidly increases the complexity, mass and power consumption of future GNSS receivers. An elegant solution to this problem is to use a technique called Direct RF Sampling, or DRFS, as described in Figure 2 and in [7]. This technique eliminates the analog frequency down-conversion, but instead performs a direct sampling of a wide frequency bandwidth from the RF spectrum and converts the useful information to baseband frequency in the digital domain. The frequency down-conversion is realized with digital circuits using an FPGA (Field Programmable Gate Array), for instance. Thus, by carefully selecting the sampling frequency, one can sample many signals simultaneously with a single RF front-end. Note that this approach also has drawbacks, such as the need for a higher sampling frequency with higher power consumption, a larger sampled bandwidth leading to faster digital processing, and more sensitivity to sampling jitter. No published work presents a real-time processing architecture tightly coupled with a GNSS receiver. We thus present for the first time a real-time DRFS prototype using commercial off-the-shelf technology to overcome the DRFS hardware obstacles, including the analog-to-digital converter (ADC). 


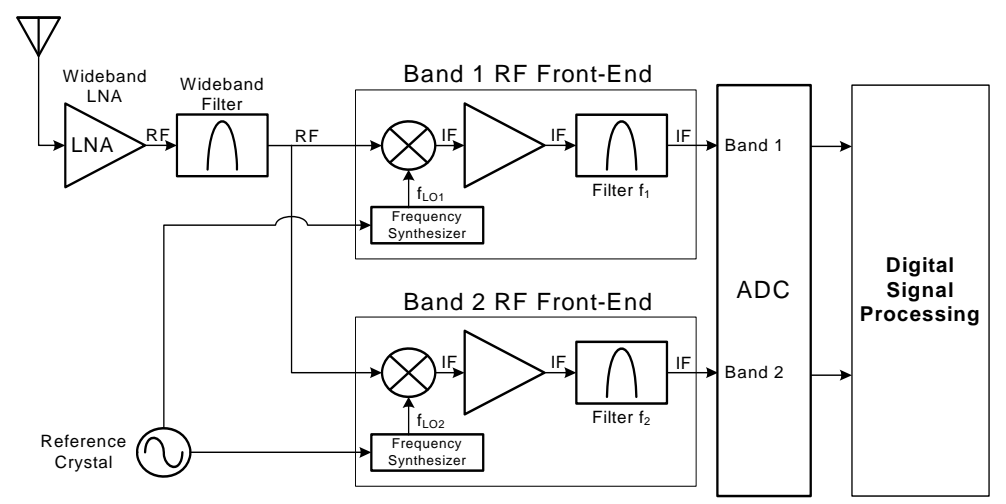

Figure 1. Superheterodyne multi-band GNSS receiver architecture.

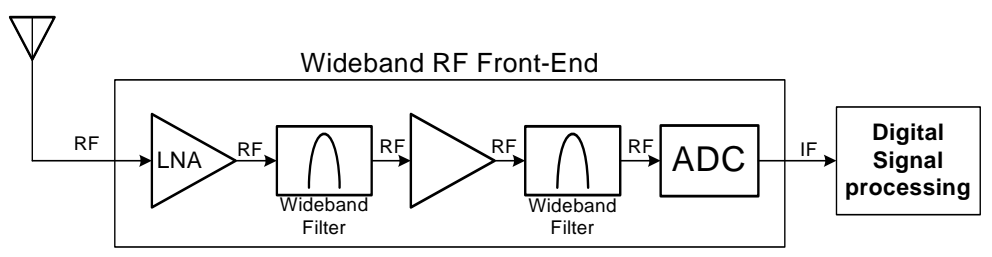

Figure 2. DRFS multi-band GNSS front-end architecture.

This first DRFS prototype is limited to the higher end of the GNSS spectrum, which contains the widely used GPS L1 Coarse Acquisition (C/A) signal as well as current and future signals from other constellations as shown in Table 1. The full band of interest for all the signals in Table 1 spans from $1559.05 \mathrm{MHz}$ to 1609.59 $\mathrm{MHz}$, thus covering a bandwidth of $50.54 \mathrm{MHz}$. For the purpose of this article, it is referred hereafter to as the G1 band. Table 2 contains all the remaining GNSS bands either currently broadcasted or expected to be in the near future. This second band, named G2-5, spans from 1164.45 MHz to $1299.21 \mathrm{MHz}$, yielding a total bandwidth of $134.76 \mathrm{MHz}$. The ultimate goal of the proposed prototype is to capture both the G1 and the G2-5 bands and thus gain access to all the navigation signals at once.

\subsection{Band-Pass Sampling}

When an up-converted frequency band is sampled, the sampling process is called band-pass sampling [13]. In fact, when multiplied by the sampling clock signal, the information bandwidth is replicated (through the aliasing principle) at integer multiples of the clock fundamental frequency $\left(f_{s}\right)$, thereby theoretically creating an infinite number of replicas of the information band in the frequency domain. Assuming a proper choice of the sampling frequency, one of these replica falls within a bandwidth below the sampling clock frequency divided by 2 $\left(f_{s} / 2\right)$, and therefore is effectively being down-converted to an intermediate frequency $\left(f_{I F}\right)$ below $f_{s} / 2$. Through this sampling process, this replica is also converted from the analog domain to the digital domain by the ADC.
Table 1. Present and future GNSS signals in the G1 band.

\begin{tabular}{cccc}
\hline GNSS & $\begin{array}{c}\text { Band } \\
\text { Name }\end{array}$ & Center Frequency & $\begin{array}{l}\text { Full Signal } \\
\text { Bandwidth }\end{array}$ \\
\hline GPS & L1 & $1575.42 \mathrm{MHz}$ & $24.0 \mathrm{MHz}$ \\
\hline GLONASS & L1 & $1603.6875 \mathrm{MHz}$ & $11.8125 \mathrm{MHz}$ \\
\hline Galileo & E1 & $1575.42 \mathrm{MHz}$ & $32.736 \mathrm{MHz}$ \\
\hline \multirow{2}{*}{ Compass } & B1 & $1561.098 \mathrm{MHz}$ & $4.092 \mathrm{MHz}$ \\
& B1-2 & $1589.742 \mathrm{MHz}$ & $4.092 \mathrm{MHz}$ \\
\hline
\end{tabular}

Table 2. Present and future GNSS signals in the G2-5 band.

\begin{tabular}{cccc}
\hline GNSS & Band Name & Center Frequency & $\begin{array}{c}\text { Full Signal } \\
\text { Bandwidth }\end{array}$ \\
\hline \multirow{2}{*}{ GPS } & L2 & $1227.600 \mathrm{MHz}$ & $24.0 \mathrm{MHz}$ \\
& L5 & $1176.450 \mathrm{MHz}$ & $24.0 \mathrm{MHz}$ \\
\hline \multirow{3}{*}{ GLONASS } & L2 & $1246.000 \mathrm{MHz}$ & $6.125 \mathrm{MHz}$ \\
& L3 & $1202.250 \mathrm{MHz}$ & $24.0 \mathrm{MHz}$ \\
& L5 & $1176.450 \mathrm{MHz}$ & $16.38 \mathrm{MHz}$ \\
\hline \multirow{5}{*}{ Galileo } & E5a & $1176.450 \mathrm{MHz}$ & $25.575 \mathrm{MHz}$ \\
& E5b & $1202.025 \mathrm{MHz}$ & $25.575 \mathrm{MHz}$ \\
& E6 & $1278.75 \mathrm{MHz}$ & $40.92 \mathrm{MHz}$ \\
\hline \multirow{6}{*}{ Compass } & B2 & $1207.140 \mathrm{MHz}$ & $20.46 \mathrm{MHz}$ \\
& B2-altBOC & $1191.795 \mathrm{MHz}$ & $30.69 \mathrm{MHz}$ \\
& $\begin{array}{c}\text { B3-BOC } \\
(15,2.5)\end{array}$ & $1268.520 \mathrm{MHz}$ & $35.805 \mathrm{MHz}$ \\
& L5 & $1176.450 \mathrm{MHz}$ & $24.0 \mathrm{MHz}$ \\
\cline { 2 - 4 } & & &
\end{tabular}


However, care must be taken in the choice of the sampling frequency in order to avoid overlapping negative and positive replicas of the information bandwidth. Bandpass sampling being highly non-linear, the process of finding a valid sampling frequency may be time consuming. Many articles describe algorithms that calculate the minimum sampling frequency for an arbitrary information band such as [8,14-16].

In [17], Choe and Kim propose a programmable algorithm to find a valid sampling frequency for a variable number of non-contiguous information bands. We have implemented this algorithm in a Matlab $^{\mathrm{TM}}$ function to calculate all the valid frequency ranges that are appropriate for the sampling of the G1 and the G2-5 bands. The following criteria were also considered for the sampling frequency $\left(f_{s}\right)$ selection:

1) $f_{s}>200 \mathrm{MHz}$ (the minimum clock frequency of the ADC),

2) $f_{s}=\mathrm{n} \times f_{\mathrm{RxGNSS}}$, where $f_{\mathrm{RxGNSS}}$ is the sampling frequency currently used in the RxGNSS and $\mathrm{n}$ is an integer. The goal is to keep any selected $f_{s}$ value being a multiple of the RxGNSS sampling frequency already in use in order to simplify the decimation process.

When considering the G1 band only, the algorithm finds many valid sampling frequency ranges but the first one meeting the two criteria above is $300 \mathrm{MHz}$. This is the sampling frequency used in the present DRFS prototype. If both the G1 and the G2-5 bands are considered together, there is no valid sampling frequency lower than approximately $1073 \mathrm{MHz}$. The two constraints above then bring this frequency up to $1080 \mathrm{MHz}$. This high sampling frequency represents an important challenge for the DRFS front-end design since both the ADC and the digital signal processing that follows need to cope with very high speed digital signals. Firstly, the ADC samples the signal at a much higher frequency, resulting in a significant increase in terms of jitter sensitivity as described in Section 3, thus leading to tighter requirements for the overall system jitter. Secondly, the digital signal processing design must take into account both a greater quantity of signals to process and a higher sampling rate for each of them. Indeed, the digital down-conversion and filtering chain, which is described in Section 5, has to be duplicated for each of the center frequencies listed in Table 1 and Table 2. Finally, the power consumption related to such a system is significantly higher than that of the proposed prototype running at $300 \mathrm{MHz}$. For these reasons, the first DRFS front-end prototype is designed only for the G1 band to demonstrate the concept and is a first step towards a multi-band GNSS software receiver.

This being said, to reduce the required sampling frequency, the RF front-end architecture could be modified to allow quadrature bandpass sampling [18]. Indeed, Dempster [18] recently demonstrated that implementing such a sampling scheme could lead to a sampling frequency reduction of up to $9 \%$ when compared to the standard bandpass sampling architecture. More importantly, his method provides up to four times more valid sampling frequencies [18]. The latter result is of prime interest for practical implementation of a DRFS system since, as described above, other engineering related constraints have to be taken into account when selecting the sampling frequency.

Focusing on the $300 \mathrm{MHz}$ prototype, it may be shown in [17] that the down-converted information replica that falls below $f_{s} / 2$ is centered at the inFtermediate frequency $\mathrm{f}_{\mathrm{IF}}$ determined by $f_{I F}=f_{c}-m \cdot f_{s}$, with $f_{c}$ being the center frequency of the RF signal prior to sampling and $m$ being a coefficient that represents the number of information replicas between $f_{I F}$ and $f_{c}$ inclusively. For example, with the bandpass sampling of the GPS L1 band (centered at $1575.42 \mathrm{MHz}$ ) using a $300 \mathrm{MHz}$ sampling frequency, the $\mathrm{RF}$ signal as seen by the ADC is the $5^{\text {th }}$ replica of the down-converted signal, thus implying a down-conversion of the RF signal's carrier center frequency by a shift of 5 $\times 300 \mathrm{MHz}$, i.e. down to $75.42 \mathrm{MHz}$.

\section{Jitter Effects and Analysis}

The use of a very high sampling frequency allows the analog to digital conversion of a very wide bandwidth. However, the drawback is a high sensitivity to sampling jitter, or phase noise if considered in the frequency domain, which is a critical aspect in satellite positioning techniques. In the time domain, sampling jitter is a measure describing the random variation of the period of a periodic signal over time. Indeed, there is an unavoidable uncertainty in the exact moment when a periodic signal will start another cycle.

In a system using analog-to-digital conversion, jitter arises from two sources: the sampling clock and the ADC itself. The sampling clock is usually supplied by a Phase Locked Loop (PLL) circuit and both the reference oscillator and the PLL design parameters (division factors) are responsible for the sampling clock jitter [19]. Within the ADC, thermal noise is responsible for generating extra jitter and limiting the number of usable bits of the ADC [20]. The jitter from the two different sources are combined in a Root Summed Square (RSS) fashion (since they have independent Gaussian distributions), resulting in the total jitter for the system. Practically, it is not possible to distinguish between the effects of the source jitter and those of the receiver jitter; one can only observe the combined effects.

The total jitter is measured in seconds and may also be characterized by a power spectral density centered around the clock's fundamental frequency. For this reason, when described in the frequency domain, jitter is 
commonly referred to as phase noise. Details on exact conversion from jitter to phase noise can be found in [11].

The effect of jitter on a sampled signal is the addition of noise to the sampled signal, thus reducing its Signalto-Noise Ratio (SNR). This is particularly important in a DRFS system for GNSS signals, since the high sampled frequencies (in the L-band portion of the electro-magnetic spectrum) render the SNR more susceptible to this jitter effect, as will be demonstrated below. The mathematical model itself was developed by [12] and is summarized here to provide a better understanding of the experimental results presented in Section 6 .

For a Binary Phase Shift Keying signal, like the one generated by the GPS satellites for the GPA L1 C/A signal, the noise power generated by the jitter can be calculated using (1) as in [12], namely:

$$
N_{\tau}=A^{2}\left(\frac{\sqrt{2}}{\sqrt{\pi}} \sigma_{\tau} f_{d}+2 \pi^{2} f_{c}^{2} \sigma_{\tau}^{2}\right)
$$

where $N_{\tau}$ is the noise power generated by the jitter, $f_{c}$ is the carrier frequency of the GNSS signal at ADC input, $f_{d}$ is the BPSK data frequency, $\sigma_{\tau}$ is the total system jitter and $\mathrm{A}$ is the constant carrier amplitude of the incoming BPSK signal at the ADC input. Equation (1) clearly shows that a higher sampling frequency will result in a higher noise power generated by the jitter.

Using the jitter as the only noise source, one can calculate the Signal to Jitter Ratio (SJR) of the sampled signal. The total signal power $(S)$ is considered to be the signal's carrier power $\left(A^{2} / 2\right)$ while the noise power is expressed by $N_{\tau}$ from (1), leading to the following expression for the SJR:

$$
S J R=\frac{S}{N_{\tau}}=\frac{1}{2\left(\frac{\sqrt{2}}{\sqrt{\pi}} \sigma_{\tau} f_{d}+2 \pi^{2} f_{c}^{2} \sigma_{\tau}^{2}\right)}
$$

To constrain the power of the noise generated by the jitter, [12] proposes to limit the jitter noise power to 10 $\mathrm{dB}$ below that of the thermal noise $\left(N_{t h}\right)$ so that its effects can be neglected. This constraint is written as follows:

$$
S J R \geq 10 \frac{S}{N_{t h}}
$$

According to [10], jitter will have more effect on the channel that conveys the GNSS data itself (as opposed, for example, to the dataless channel that conveys a pilot signal). Hence, a noise bandwidth $B_{d}$, equal to that of the GNSS signal (i.e. twice that of the GNSS data, given the double sideband spectrum), is used to calculate the thermal noise power (yielding $k T B_{d}[12]$ ). Using the SJR definition of (2) and solving for $\sigma_{\tau}$, the jitter constraint is expressed by:

$$
\sigma_{\tau} \leq \frac{\sqrt{\frac{80 f_{d}^{2} S}{\pi k T B_{d}}+16 \pi^{2} f_{c}^{2}}-\sqrt{\frac{80 f_{d}^{2} S}{\pi k T B_{d}}}}{8 \pi^{2} f_{c}^{2} \sqrt{\frac{10 S}{k T B_{d}}}}
$$

where $k$ is the Bolztmann constant $\left(1.38 \times 10^{-23} \mathrm{~J} \cdot \mathrm{K}^{-1}\right)$ and $T$ is the noise temperature of the GNSS receiver.

The constraint given by (4) can be considered as a design parameter for a GNSS DRFS receiver. As a numerical example, the values presented in Table 3 are used in (4) to calculate the maximum allowable jitter for the GPS L1 C/A signal. The result of this calculation is a jitter limit of $1.72 \mathrm{ps}$.

However, it is yet to be proven experimentally that the jitter limit expressed by (4) (i.e. $1.72 \mathrm{ps}$ in this numerical example) actually results in the jitter having no contribution to the SNR of the GNSS signal. The DRFS front-end described in this article was designed for a significantly lower total jitter in order to have a good reference in assessing the effects of intentionally degraded sampling jitter on the GNSS signals. A critical part of the proposed architecture is the analog-to-digital converter (ADC) and therefore a high frequency ADC from Atmel, the AT84AS004, was selected for this function because of its very low jitter $(0.15 \mathrm{ps})$. The DRFS prototype was tested and analyzed in 2009 and presented in [21]. The signal's demodulation and the data processing that follows are realized within the RxGNSS, which uses the Lyrtech VHS-ADC development board. This board, connected to a Personal Computer (PC) through a Compact PCI bus, includes a Virtex 4 FPGA from Xilinx as well as analog-to-digital converters and digital input/output interfaces. The GNSS data processing implemented in the RxGNSS was developed at the LACIME laboratory in the form of a Software Defined Radio. The FPGA demodulates 12 GPS channels in parallel with a $60 \mathrm{MHz}$ clock frequency. A Microblaze microcontroller, from Xilinx, then proceeds with the acquisition and tracking of the signals in real-time using early, prompt and late correlators and the classical phase and delay locked loops each of which using a numerically controlled oscillator.

Table 3. GPS L1 C/A parameters for maximum jitter constraint.

\begin{tabular}{ccc}
\hline Parameter & Description & Value \\
\hline$S$ & Carrier power & $-158.5 \mathrm{dBW}$ \\
$T$ & Reference noise temperature & $290 \mathrm{~K}$ \\
$f_{c}$ & Carrier nominal frequency & $1575.42 \mathrm{MHz}$ \\
$f_{d}$ & Pseudo Random Noise (PRN) code rate & $1.023 \mathrm{MHz}$ \\
$B_{d}$ & Data bandwidth & $100 \mathrm{~Hz}$ \\
\hline
\end{tabular}


The architecture itself is similar to the one presented in [22] and is presented in detail in [4].

The GNSS signal processing in the RxGNSS is constantly evolving to provide better performances and to support new signals and modulation schemes with the help of a recently patented universal GNSS tracking channel $[23,24]$.

\section{RF Design Description}

The design of a DRFS front-end for GNSS signals is very challenging for various reasons. At the user level altitude, GNSS signals have a power below that of the thermal noise ( $-158.5 \mathrm{dBW}$ for the GPS L1 C/A signal) and the consequences of this are twofold. First, it implies that the signals are more sensitive to the noise generated by the receivers, so it is mandatory to keep the receiver's noise figure as low as possible.

Second, for the sampled signal amplitude to cover the full input range of the $\mathrm{ADC}$, the gain required is on the order of $100 \mathrm{~dB}$. This high gain can only be achieved by cascading multiple amplifiers, which can lead to instability. Filtering is another challenging topic in DRFS receiver design. The architecture allows sampling over a very wide bandwidth, and unwanted signals outside that bandwidth might be aliased onto the desired signals through the sampling process. Therefore, wideband filters with good rejection and low insertion loss are required.

The DRFS prototype was designed with the future objective of capturing all the GNSS signals in L-Band. In fact, all the components have been selected to achieve this long term objective except for the filters, which for this initial study were chosen to be narrowband centered on the GPS L1 frequency. Hence, only the GPS L1 C/A signal was tested in real-time. The complete architecture of the DRFS prototype is shown in Figure 3 with the parts identified.

The GPS L1 C/A signal is first captured by a GPS704X NovAtel antenna before being amplified by a 30 $\mathrm{dB}$ wideband gain Low Noise Amplifier (LNA) from Hittite, having a $1.3 \mathrm{~dB}$ noise figure. Then, the signal passes through a series of wideband Gain Block Amplifiers (GBA) and narrowband filters to reject out-of-band signals. Each of the GBA (from Avago Technologies) provides $22 \mathrm{~dB}$ of gain from $\mathrm{DC}$ to $3.5 \mathrm{GHz}$. The filters (from RFM Inc.) have a $15.3 \mathrm{MHz}$ bandwidth $(3 \mathrm{~dB})$ and a center frequency equal to that of the GPS L1 C/A signal, i.e. $1575.42 \mathrm{MHz}$. Although this $3 \mathrm{~dB}$ cut-off bandwidth is narrower that the $20.46 \mathrm{MHz}$ full GPS information band (Table 1), it is appropriate for the purpose of our experiments, since most of the GPS L1 information is concentrated within a $2.046 \mathrm{MHz}$ band across the center frequency [22].

The last amplifier is a wideband Variable Gain Amplifier (VGA) (from Hittite) with a gain that can be set through digital control lines from $-13.5 \mathrm{~dB}$ to $+18 \mathrm{~dB}$ in steps of $0.5 \mathrm{~dB}$. The VGA is required to adjust the power level at the input of the ADC so that it is not overrun while keeping a good bit resolution on the signal.

The ADC (the AT84AS004 from Atmel) then samples the GPS L1 C/A signal at a rate of $300 \mathrm{MHz}$ (according to a band-pass sampling scheme) before transferring the digitalized data in parallel to an FPGA. More details on the digital design of the FPGA are provided in Section 5.

The various amplification, filtering and gain control building blocks were soldered on individual RO3006

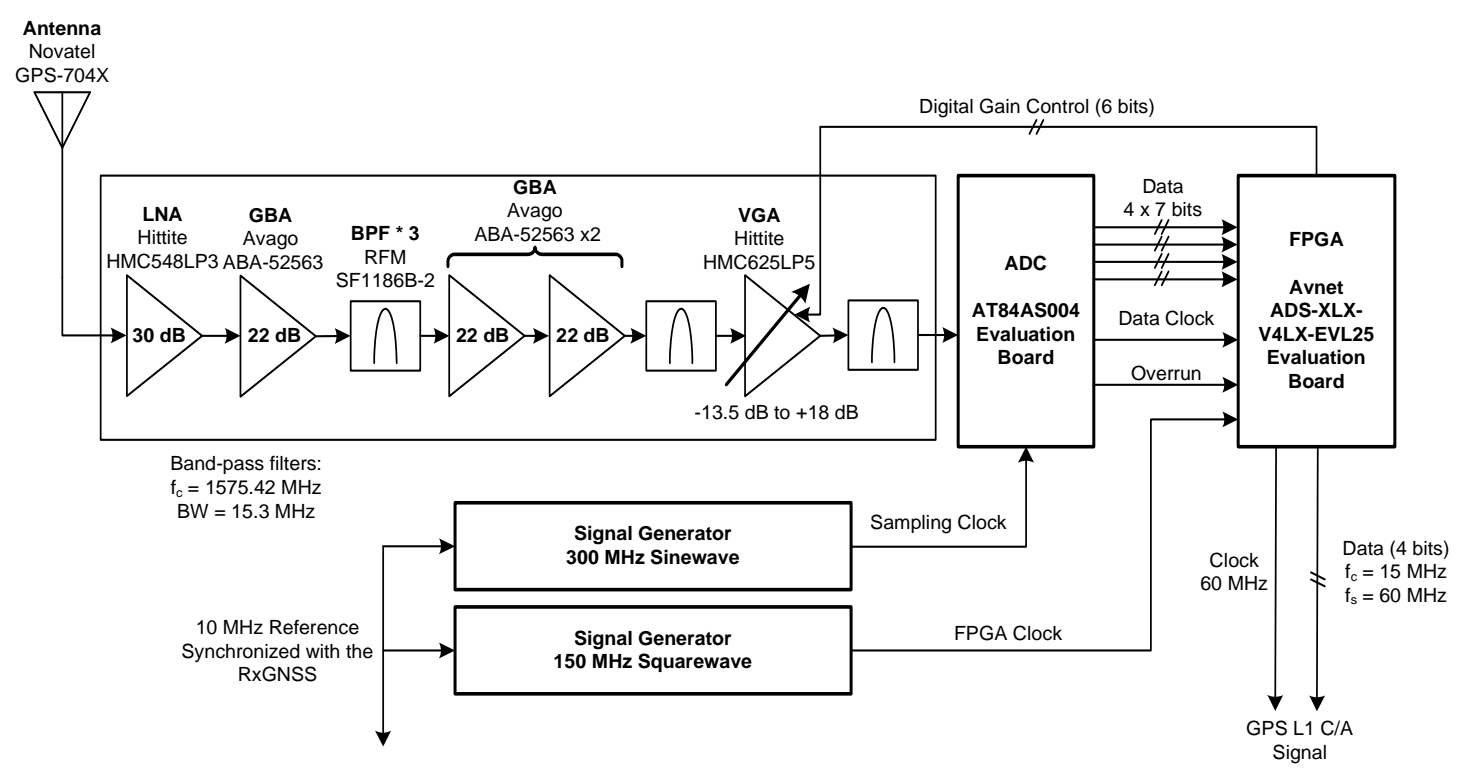

Figure 3. DRFS prototype architecture. 
substrates (from Rogers Corporation) along with matching and power supply circuits. The high gain of the amplification chain can result in feedback from one amplifier to another and then lead to instability of the whole amplification chain. In the current design, a spatial isolation between the various amplifiers is realized by packaging them individually in separate aluminum casings with SMA connectors. This RF front-end is thus heavier and bulkier, but this approach eliminates any radiating and conducting interference. Figure 4 shows the LNA in its aluminum casing, as an illustrative example. Future work on the prototype includes the implementation of the amplification chain on a single RF substrate, which will require a careful design to avoid feedback between the amplifiers. The use of attenuators, or even isolators, between the amplification stages as well as filtering of the power circuitry will be considered.

Each building block was fabricated and its scattering parameters were measured. Using the Advanced Design System (ADS ${ }^{\mathrm{TM}}$ ) circuit simulator, a model of RF frontend was built where each block was represented by its measured parameters. The simulated model shows that, from the LNA input to the output of the last filter, the total gain can be adjusted between $71.4 \mathrm{~dB}$ and $102.0 \mathrm{~dB}$ with a noise figure of $1.52 \mathrm{~dB}$ (at maximum gain). The degradation of the noise figure from $1.3 \mathrm{~dB}$ (the LNA alone) to $1.52 \mathrm{~dB}$ is first due to the losses from the LNA assembly input to the chip itself (connector, trans- mission line and matching circuit). The noise figure of the GBA (3.5 dB), the filters (3.0 dB) and the VGA $(6.75 \mathrm{~dB})$ contribute very slightly to the total noise figure since the LNA has a high $30 \mathrm{~dB}$ gain. Figure 5 shows the result of the simulation.

The limitation of the current architecture to the GPS L1 signal is a result of the band-pass filters. A flexible architecture would require more complex filters in order to keep the G1 and the G2-5 GNSS bands while filtering the out-of-band noise between them. This could be realized through a wide band-pass filter in cascade with stop-band filters. Another approach would be the use of two diplexers; one to split and filter the two main GNSS bands and the other one to recombine them. None of these filters are currently available off-the-shelf and they both present serious design challenges that are beyond

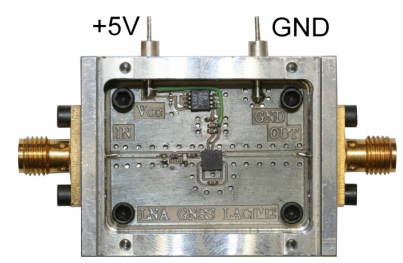

Figure 4. Sample of the aluminum casing showing the LNA assembly. the scope of the proposed architecture at this stage. In addition, both designs would also have a higher insertion loss, leading to a potential lower sensitivity of the GNSS receiver.

\section{Digital Signal Processing Design Description}

The ADC (in Figure 3) samples the signal at $300 \mathrm{MHz}$, which is appropriate for the band-pass sampling of the 20.46 MHz bandwidth GPS L1 C/A signal, as explained in Section 2. Furthermore, the RxGNSS receiver has some interfacing constraints that require digital signal processing before the data can be demodulated. The $\mathrm{ADC}$ and the RxGNSS (in Figure 3) data characteristics are described in Table 4.

A Virtex 4 FX 12 evaluation board was used to implement the digital signal processing in VHDL. Its maximum operating frequency is high enough to accommodate the four $75 \mathrm{MHz}$ data buses from the ADC. It has enough logic gates and Digital Signal Processor (DSP) blocks to perform the required processing, as it is demonstrated below. The design uses various DSP Cores, which can be described as building blocks in VHDL to help facilitate the design of basic functions. For instance, a First In First Out (FIFO) memory block was used in this implementation to capture the signal at various processing points within the FPGA.

Using a custom Visual Basic .NET interface, the samples are then transferred to a computer through a serial port and post-processed in Matlab to monitor the signal

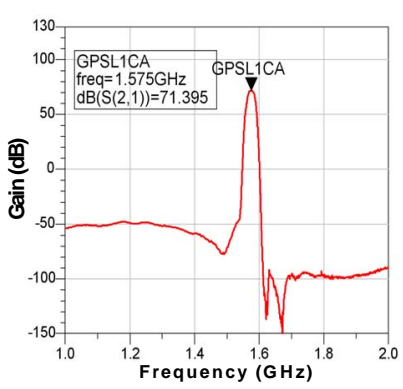

(a)

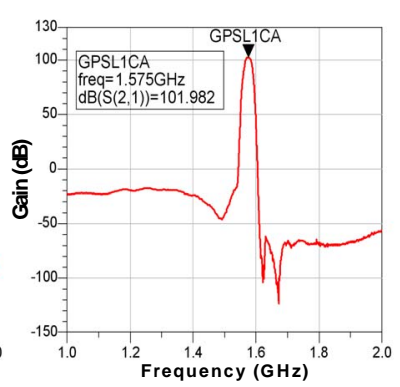

(b)
Figure 5. DRFS RF front-end gain with VGA set to minimum (a) and with VGA set to maximum (b).

Table 4. ADC and RxGNSS interfacing characteristics.

\begin{tabular}{ccc}
\hline Signal parameters & ADC & RxGNSS \\
\hline $\begin{array}{c}\text { Sampled GPS L1 C/A } \\
\text { center frequency }\end{array}$ & $f_{I F 1}=75.42 \mathrm{MHz}$ & $f_{I F 2}=15 \mathrm{MHz}$ \\
Data rate & $\begin{array}{c}f_{s 1}=300 \mathrm{MHz} \text { separated in } \\
4 \text { bus of } 75 \mathrm{MHz} \text { each } \\
\text { (A, B, C, D) }\end{array}$ & $\begin{array}{c}f_{s 2}=60 \mathrm{MHz} \\
\text { on a single bus }\end{array}$ \\
Bit resolution & 7 bits & 4 bits \\
\hline
\end{tabular}


while it progresses through the FPGA. A real GPS L1 $\mathrm{C} / \mathrm{A}$ signal acquired with this FIFO is shown in Figure 6(a). This signal is centered at $75.42 \mathrm{MHz}$.

The first step in the digital signal processing is motivated by the difference in center frequency of the GPS L1 C/A signal between the ADC and the RxGNSS. It requires a digital frequency down-conversion using a digital Local Oscillator (LO) of $f_{I F 1}-f_{I F 2}=60.42 \mathrm{MHz}$. However, the signal is split into 4 buses and the Virtex 4 FPGA is not fast enough to serialize them for the multiplication to take place. Therefore, a poly-phase digital down-conversion is implemented with four sine-wave generators (A, B, C and D) in DSP Cores, each generating their signal at a $75 \mathrm{MHz}$ sampling rate. Sine-wave generator $\mathrm{A}$ is first multiplied with the first sample on the A bus, then sine-wave generator B is phase shifted to reproduce the time difference between samples on bus $A$ and $\mathrm{B}$, and so on for the other sine-wave generators.

The resulting LO of $60.42 \mathrm{MHz}$ is presented in Figure 6(b), taken from real-time data captured in the FPGA by

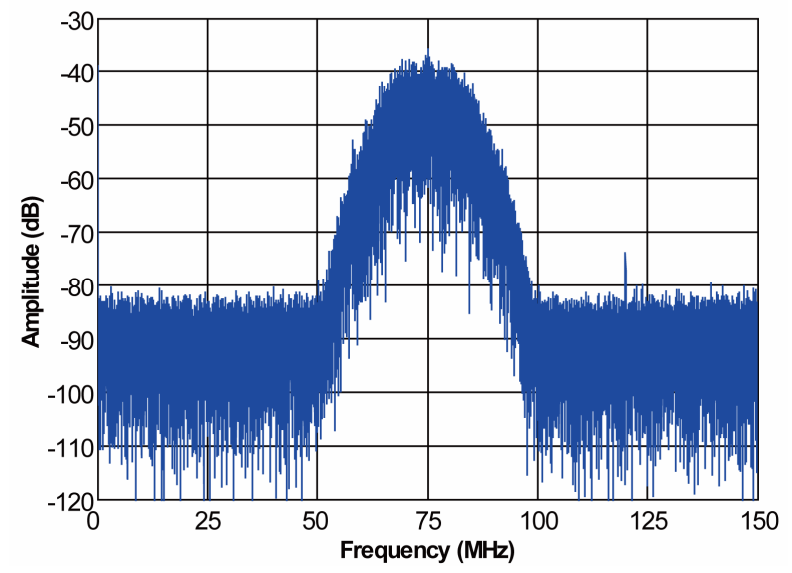

(a)

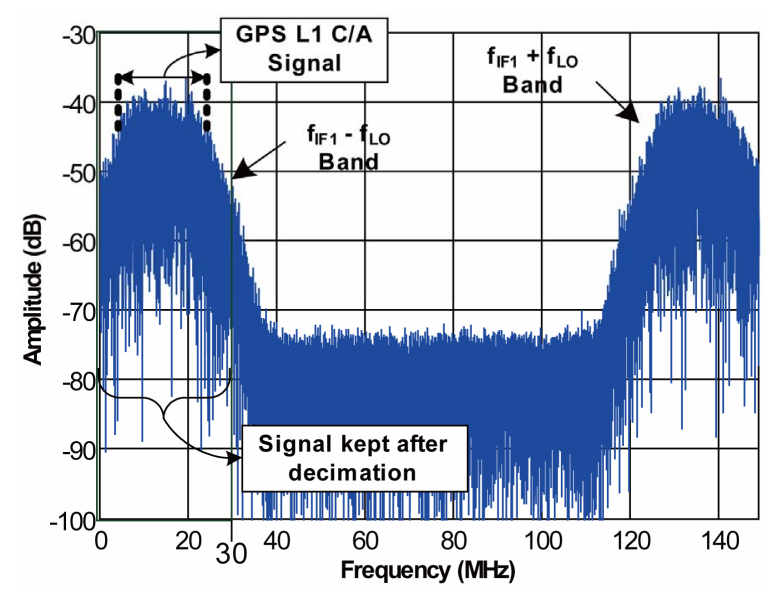

(c) the FIFO. After the frequency down-conversion, there is an image centered around $135.84 \mathrm{MHz}$ but the relevant signal occupies roughly the first $30 \mathrm{MHz}$ over the 150 $\mathrm{MHz}$ bandwidth as it can be seen in Figure 6(c).

The second step is the conversion from a $300 \mathrm{MHz}$ bus to a $60 \mathrm{MHz}$ bus. The process of reducing the sampling frequency of a signal is called decimation. However, one cannot simply disregard one sample out of 5 to get the desired output sampling rate, as it would create aliasing of the signal. In fact, some filtering needs to take place before the decimation process to reduce the aliased noise in the desired signal.

The digital filter is also used to remove the image product of the digital down-conversion (see Figure 6(c)). But it would not be efficient to filter 5 samples at 300 $\mathrm{MHz}$ and discard 4 of them. The solution is to split the signal in 5 phases, filter each of them at one fifth of the speed and sum the result to have a single sample at 60 $\mathrm{MHz}$. This process is known as a factor 5 polyphase decimation as described in [25]. A $50^{\text {th }}$ order Finite Im-

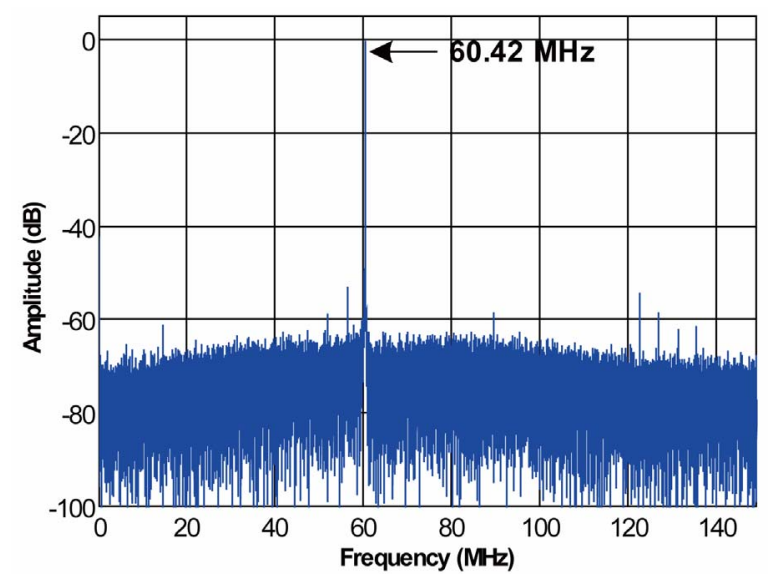

(b)

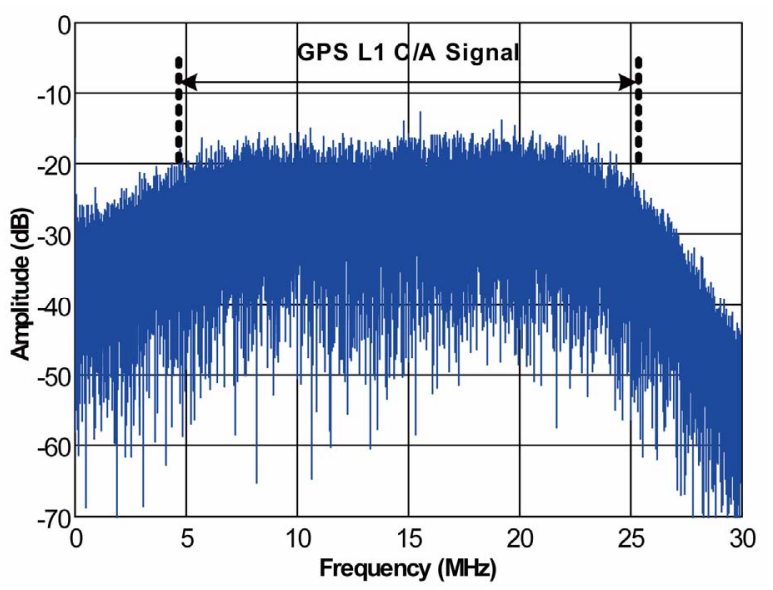

(d)

Figure 6. Real GPS L1 C/A signal as received by the FPGA (a), after frequency down-conversion (c) and after decimation (d). (b) shows the LO signal. 
pulse Response (FIR) filter was implemented for the filtering, which used most of the DSP blocks available (design optimization is still possible but was not part of this study).

A lower order filter would have been sufficient but the goal of the prototype was to reduce the noise to its lowest level. The resulting output of the polyphase decimation is presented in Figure 6(d) and the complete digital architecture is presented in Figure 7. The demultiplexer is used solely to convert the 4 buses at $75 \mathrm{MHz}$ to 5 buses at $60 \mathrm{MHz}$ (the total data rate is conserved) in order to use the factor 5 polyphase decimator.

The use of poly-phase signal processing results in a higher complexity in the digital design by duplicating every process for each signal phase and adding overhead to deal with each of those phases. For instance, the polyphase down-conversion necessitates four digital sinewave generators instead of one for a single-phase design. However, the reduction of the total number of DSP blocks due to the poly-phase filtering results, overall, in a more gate efficient design. Furthermore, the single phase signal processing, which would be at $300 \mathrm{MHz}$, would not be feasible with low grade FPGAs such as the one used for this prototype.

The implementation of the digital signal processing in an FPGA gives full flexibility to the proposed prototype. To capture the other GNSS band located in G1 and G2-5, the architecture of Figure 7 would be duplicated for each different band's center frequency. The input data would have the same source and only the sine-wave generator's frequency would be different for each center frequency so that each of the captured bands is centered around 15 $\mathrm{MHz}$ after the digital down-conversion. As calculated in Section 2, a sampling frequency of $1080 \mathrm{MHz}$ would be required to capture all the GNSS bands and this repre- sents a significant challenge in terms of digital signal processing design. The maximum clock frequency at which the FPGA can process the signals as well as the number of DSP blocks and gates available are the main limiting factors. However, FPGA performances evolve rapidly and mass market devices are readily available to achieve such challenging processing.

In addition, while the DRSF front-end is transparent to the modulation format or the code length of the various GNSS signals, the RxGNSS would also need modifications in order to demodulate and process all of the new bands.

\section{Experimental Measurement Results and Analysis}

\subsection{DRFS Prototype Validation Measurements}

In order to validate the DRFS prototype design, a functional test was performed by connecting the DRFS architecture of Figure 3 to the RxGNSS as shown in Figure 8. A real GPS signal was captured by a GPS-704X Novatel antenna (installed on the roof of the university campus) and was pre-amplified by an LNA before being subjected to losses through the long cables between the roof-top antenna and the LNA in the receiver chain of Figure 3. The signal is then equally split to feed both a Novatel DL-4 Plus receiver and the DRFS prototype. In this configuration, the Novatel receiver is used to confirm the presence of the GPS signal for the duration of the test and to compare the performance of the two architectures. The interconnecting circuit is a simple passive printed circuit board (PCB) used to connect the ADC output ports to the right input ports of the FPGA. A Symmetricom 8040 rubidium $10 \mathrm{MHz}$ frequency reference is used for both the DRFS prototype and the RxGNSS in order to

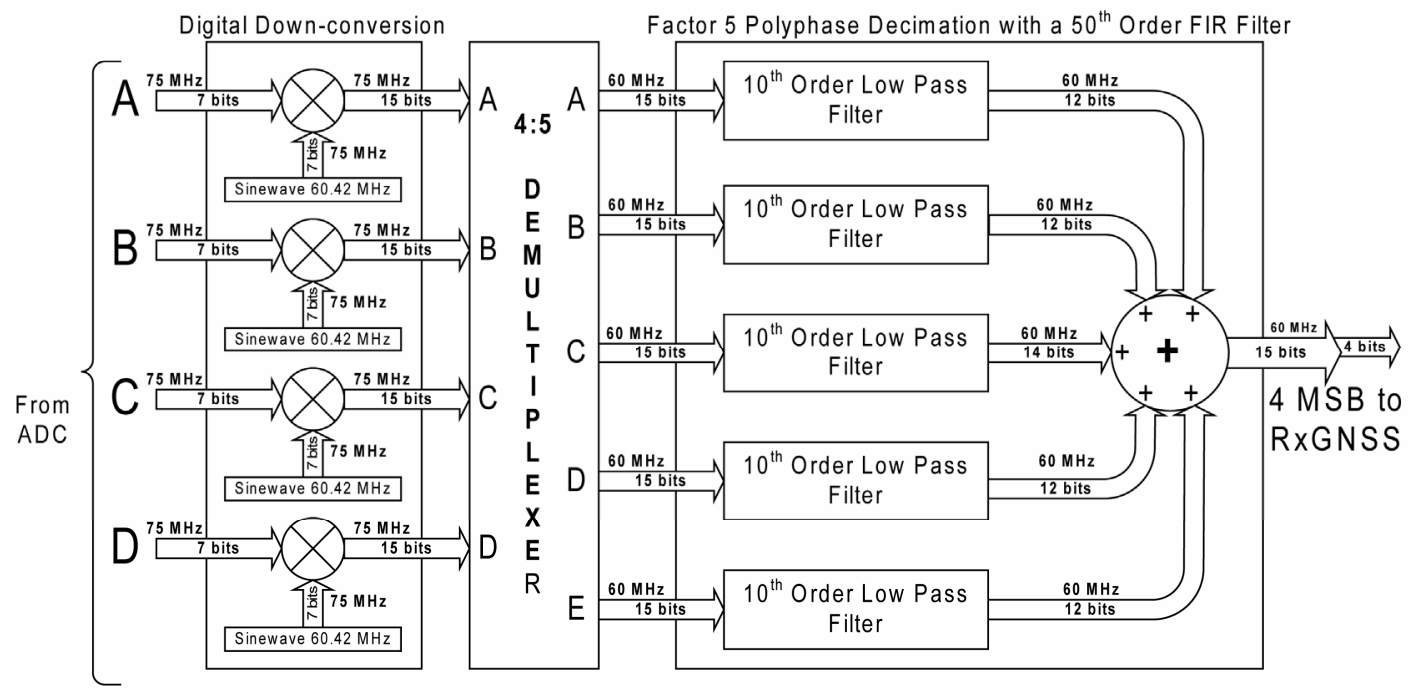

Figure 7. Validation test bench of the DRFS receiver. 


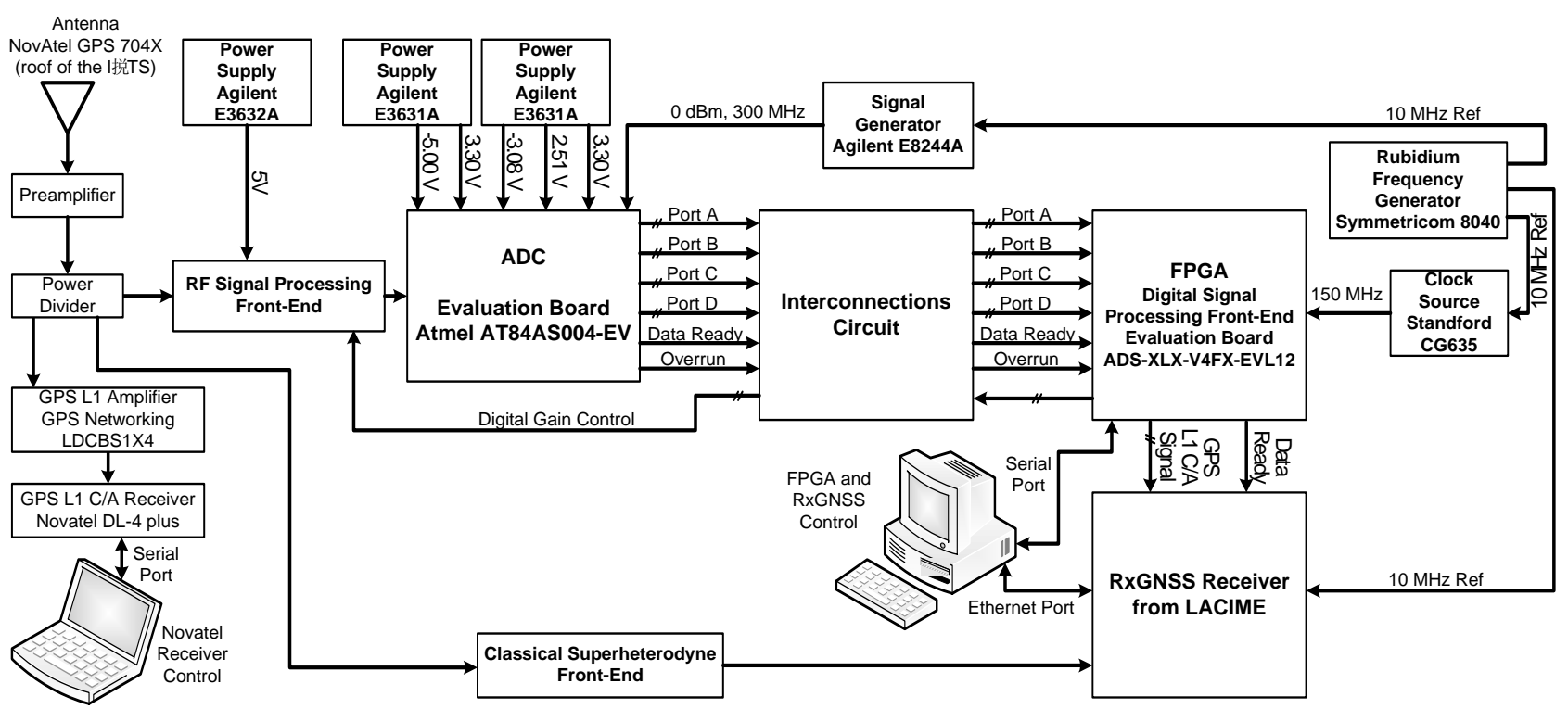

Figure 8. Digital signal processing architecture in the FPGA of the DRFS.

eliminate the clock drift between the two. Three power supplies generate the various voltages required to power the RF amplification chain and the ADC.

The position of the roof-top antenna was previously determined precisely using the Novatel DL-4 plus receiver over a long period of time, yielding the values presented in Table 5.

For the validation test, the RxGNSS recorded more than 15 hours of the Position, Velocity and Time (PVT) solution. This data was then compared with previously acquired data from the RxGNSS using a conventional superheterodyne front-end. The conventional front-end used an analog LO derived from a $10 \mathrm{MHz}$ reference signal with slightly better phase noise than the one used for the DRFS test. Figure 9 illustrates the two dimensional position in terms of North-South error and EastWest error for both front-ends while Table 6 groups the average and the standard deviation of the various errors.

The horizontal error is calculated trigonometrically using the well-known square root of the sum of the square of the North-South and East-West errors. The difference is calculated by subtracting the standard deviation of the superheterodyne front-end from the one of the DRFS front-end and dividing the result by the standard deviation of the superheterodyne front-end, which is

Table 5. Coordinates of the novatel GPS-704x antenna installed on the roof.

\begin{tabular}{cc}
\hline Coordinate & Value \\
\hline Latitude & $45.494042129196487^{\circ}$ North \\
Longitude & $73.562788220317856^{\circ}$ West \\
Altitude & $41.719675601984839 \mathrm{~m}$ \\
\hline
\end{tabular}

thus considered as the reference.

Based on the results presented in Table 6, the accuracy of the DRFS front-end is slightly better than that of the conventional superheterodyne front-end. The improvement is in the order of $14 \%$ for the standard deviation of the horizontal error. Those results are more perti-

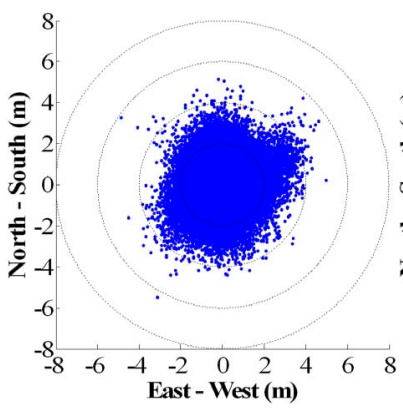

(a)

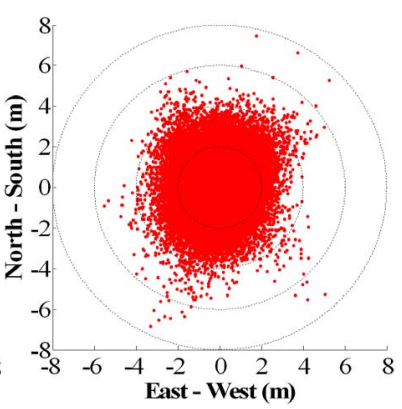

(b)
Figure 9. Validation test: DRFS (a) and superheterodyne (b) front-end relative position errors. The relative position error is the difference in meters, on a bidimensionnal surface, between the position measured by the front-end under test and the reference position of the antenna given in Table 5.

Table 6. Validation test: RxGNSS relative position error.

\begin{tabular}{cccc}
\hline & $\begin{array}{c}\text { Superheterodyne } \\
\text { front-end }\end{array}$ & \multicolumn{2}{c}{ DRFS front-end } \\
\hline Error Type & Std (m) & Std (m) & Difference (\%) \\
North-South & 1.259 & 1.104 & -12.31 \\
East-West & 1.059 & 0.992 & -6.33 \\
Altitude & 2.366 & 2.253 & -4.78 \\
Horizontal & 0.879 & 0.753 & -14.33 \\
\hline
\end{tabular}


ment in the context of measuring a trend in relative terms, rather than absolute terms, considering the fact that the two front-ends are difficult to compare because of their significantly different architecture. Nevertheless, our validation test demonstrated that a DRFS front-end can provide similar position accuracy when compared to a conventional superheterodyne front-end.

\subsection{Jitter Effects Measurements and Analysis}

The second part of the testing aims at verifying the assumption made in Section 3 regarding the maximum sampling jitter of a DRFS architecture. For the GPS L1 C/A signal, the maximum jitter was calculated using the parameters in Table 3 and found to be 1.72 ps. However, a proper real time test has to be performed to ensure this limit is sufficient. For this purpose, the test bench of Figure 10 was used. This bench is similar to the one used for the validation test except for two aspects.

First, the ADC clock's phase noise is made adjustable by combining the $300 \mathrm{MHz}$ sampling clock signal with Gaussian noise signal generated by a wideband noise source (NoiseCom NC 6108). The noise power is set manually with attenuators having insertion losses from 0 $\mathrm{dB}$ to $60 \mathrm{~dB}$. The equivalent jitter of each of these attenuation steps is calibrated with the Agilent E5052 signal source analyzer, which is capable of measuring noise power around a carrier from $1 \mathrm{~Hz}$ to $40 \mathrm{MHz}$. This technique that uses a noise source is similar to the one described in [26]. However, instead of using a phase modulator, a simple RF power combiner adds wideband voltage noise on the clock signal, effectively adding noise power density around its center frequency.

Thus, the twelve different sampling jitters values listed in Table 7 were used in this analysis of the jitter effects. It should be noted that the $33 \mathrm{~dB}$ attenuation corresponds to the jitter that is closest to the $1.72 \mathrm{ps} \mathrm{limit.} \mathrm{Also,} \mathrm{the}$ $\mathrm{ADC}$ intrinsic jitter $(0.15 \mathrm{ps})$ is included in those values.

Second, instead of capturing a real GPS signal with an antenna, the GPS simulator STR4500 from Spirent simulates a GPS signal with the characteristics specified in the different tests below.

\subsubsection{First Jitter Effects Verification: Correlation Peak Measurements}

The first jitter effect verification was performed using only the DRFS prototype (i.e. without connecting the RxGNSS) by capturing the data in the FIFO imple-

Table 7. Noise source attenuation and corresponding clock jitter.

\begin{tabular}{cc}
\hline Noise Source Attenuation & $\begin{array}{c}\text { Corresponding total sampling } \\
\text { jitter (s) }\end{array}$ \\
\hline Noise Source OFF (Reference) & 0.243 \\
45 & 0.532 \\
38 & 1.044 \\
33 & $\mathbf{1 . 8 3 9}$ \\
32 & 2.062 \\
24 & 5.033 \\
18 & 10.54 \\
8 & 21.11 \\
7 & 30.04 \\
6 & 34.01 \\
5 & 38.20 \\
\hline
\end{tabular}

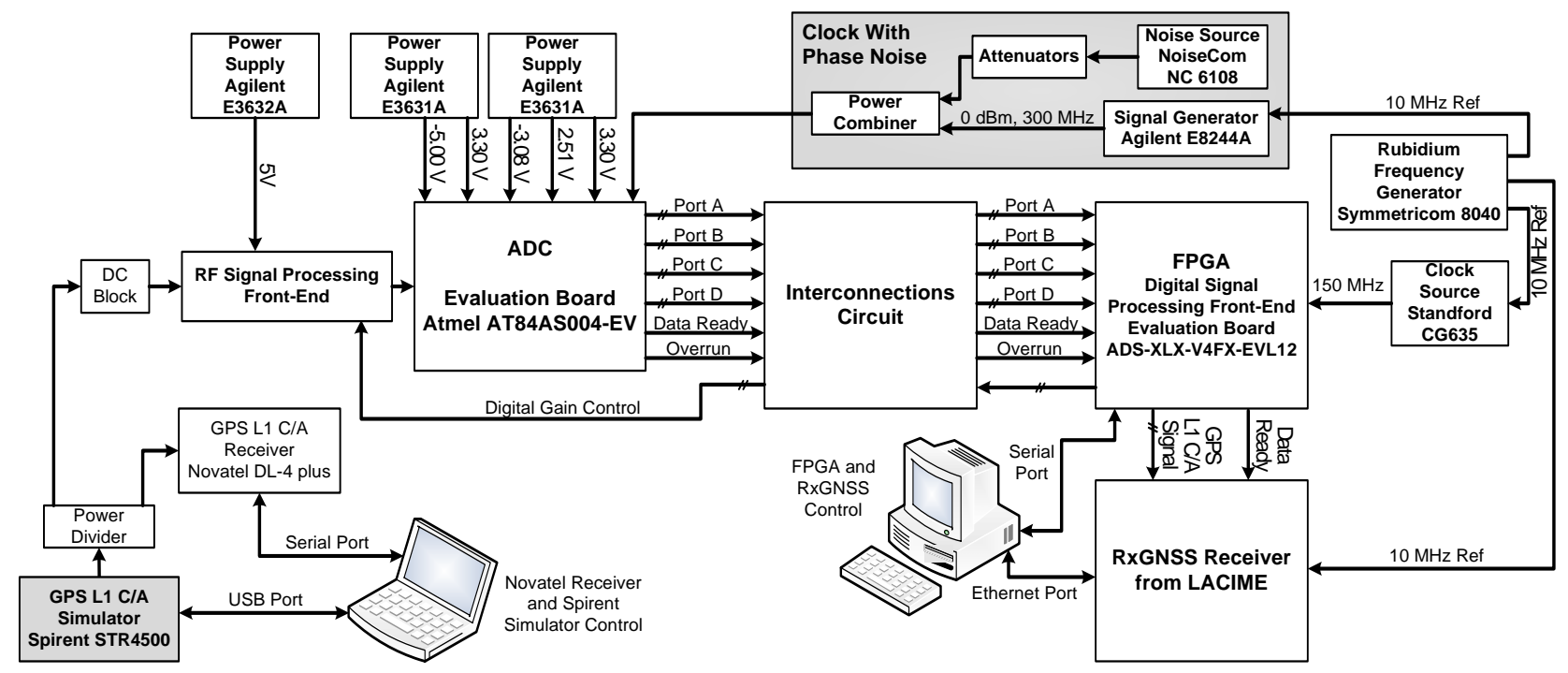

Figure 10. Jitter effects test bench of the DRFS receiver. 
mented within the FPGA as described in Section 5. In this test, the effect of the sampling clock jitter alone on the DRFS prototype (i.e. excluding any other source of performance degradation) is evaluated by observing the loss in the amplitude of the correlation peak of the GPS L1 C/A signal. For each measurement, 2 ms of GPS signal at a sampling rate of $60 \mathrm{MHz}$ over 4 bits is transferred to the computer using a serial port. Longer data segments would have produced betters results but the FIFO cannot handle more data due the FPGA size used for the prototype. Then, Matlab ${ }^{\mathrm{TM}}$ functions were used to process the samples by performing a Fast Fourrier Transform (FFT) correlation on one PRN period and identifying the code delay leading to the highest correlation peak.

In order to observe the effect of the sampling clock jitter only, a static receiver scenario was configured in the simulator. Moreover, to avoid dealing with Doppler uncertainties and with secondary correlation peaks, a single GPS satellite was simulated in a GEO orbit at $0^{\circ}$ $\mathrm{W}$. With these parameters, only the PRN code delay is unknown when searching for the PRN's correlation peak. The carrier to noise power density ratio $\left(\mathrm{C} / \mathrm{N}_{0}\right)$ was varied from $50 \mathrm{~dB} \cdot \mathrm{Hz}$ down to $35 \mathrm{~dB} \cdot \mathrm{Hz}$ with $5 \mathrm{~dB}$ steps and the jitter was varied according to the values of Table 7 using coaxial attenuators. For each $\mathrm{C} / \mathrm{N}_{0}$ and clock jitter combination, 100 measurements of 2 ms were captured and the amplitude of the correlation peak was monitored. The 100 measurements were averaged to obtain a good statistical distribution. Table 8 and Figure 11 present the jitter effects measurement results for each $\mathrm{C} / \mathrm{N}_{0}$ and clock jitter combination in the form of a correlation peak's amplitude loss (in $\mathrm{dB}$ ) relative to the reference clock jitter (with the noise source OFF). The simulated GEO satellite orbit along with the static receiver scenario means that the GPS signal's carrier has a Doppler shift of $0 \mathrm{~Hz}$. Therefore, after the FFT correlation process, the correlation peak is located in the $0 \mathrm{~Hz}$ Doppler bin. If the highest correlation value is located in another Doppler bin, the measurement is a false detection and is due to the surrounding noise power being higher than the real correlation peak. All the false detections found in the 100 measurement are removed from the average.

The first conclusion from these results is that a sampling clock jitter lower than 5 ps does not have a significant effect on the correlation peak's amplitude. This confirms what was assumed theoretically in Section 3, i.e. when the sampling jitter is limited so as to maintain a jitter related noise that is at least $10 \mathrm{~dB}$ lower than the thermal noise, the jitter has a negligible effect on the quality of the signal detection. Therefore, when receiving the GPS L1 C/A signal, the 1.72 ps sampling jitter limit derived from (4) is sufficient, with enough margin. Be- yond the 5 ps sampling jitter point, the correlation peak's amplitude starts dropping as the sampling jitter increases (see Figure 11).

The four $\mathrm{C} / \mathrm{N}_{0}$ cases behave similarly, except for the $35 \mathrm{~dB} \cdot \mathrm{Hz}$ which has a smoother slope. The latter case is due to the amount of false detections in the 100 measurements (up to $84 \%$ depending on the sampling jitter), as can be expected with a poor $\mathrm{C} / \mathrm{N}_{0}$ condition. This means that only the highest correlation peaks are used in the average calculation, leading to an artificially higher correlation peak amplitude. The number of false detections is greater for the highest sampling jitter and the lower $\mathrm{C} / \mathrm{N}_{0}$. In fact, the $42.71 \mathrm{ps}$ sampling jitter is so high that no valid correlation peak (in the $0 \mathrm{~Hz}$ Doppler bin) is detected in more than $95 \%$ of the measurements.

\subsubsection{Second Jitter Effects Verification: Position Accuracy and $\mathbf{C} / \mathbf{N}_{0}$ Measurements}

The second jitter effect verification was performed this time with the RxGNSS connected and by simulating a true GPS constellation with a static receiver. In this test, the effect of the sampling clock jitter through the entire receiver (including the RxGNSS block of Figure 10) was evaluated by observing the error in the relative position of the receiver as well as the degradation of the $\mathrm{C} / \mathrm{N}_{0}$ as calculated by the algorithm of the RxGNSS block. For the same $\mathrm{C} / \mathrm{N}_{0}$ and sampling jitter combinations previously used, the RxGNSS recorded the PVT solution as well as a $\mathrm{C} / \mathrm{N}_{0}$ estimation for a period of one hour. It should be noted that the $\mathrm{C} / \mathrm{N}_{0}$ estimation from the RxGNSS block differed slightly from the adjusted $\mathrm{C} / \mathrm{N}_{0}$ on the Spirent simulator but since only the degradation of the $\mathrm{C} / \mathrm{N}_{0}$ is of interest, this difference has no impact on the analysis. The relative position errors and the estimated $\mathrm{C} / \mathrm{N}_{0}$ were averaged over this period and the simulation scenario was restarted for each combination. The processed results of those measurements are presented in Table 9 and Figure 12 for the horizontal error

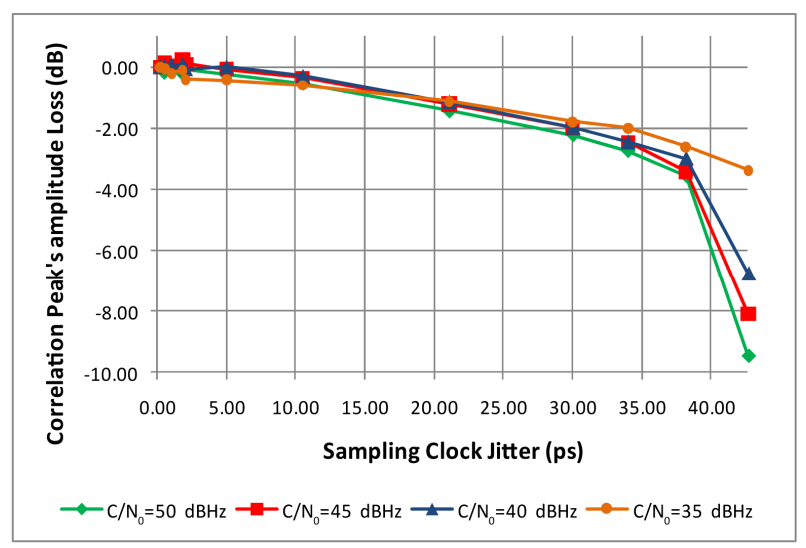

Figure 11. Clock jitter effects on the correlation peak's amplitude. 
Table 8. Sampling clock jitter effects on the correlation peak's amplitude, averaged over 100 measurements. False detections are removed from the average.

\begin{tabular}{|c|c|c|c|c|}
\hline & \multicolumn{4}{|c|}{ GPS Signal's $\mathrm{C} / \mathrm{N}_{0}$} \\
\hline & $50 \mathrm{~dB} \cdot \mathrm{Hz}$ & $45 \mathrm{~dB} \cdot \mathrm{Hz}$ & $40 \mathrm{~dB} \cdot \mathrm{Hz}$ & $35 \mathrm{~dB} \cdot \mathrm{Hz}$ \\
\hline Sampling Clock Jitter (ps) & Peak Loss (dB) & Peak Loss (dB) & Peak Loss (dB) & Peak Loss (dB) \\
\hline 0.243 & 0.000 & 0.000 & 0.000 & 0.000 \\
\hline 0.532 & -0.166 & 0.132 & 0.030 & -0.051 \\
\hline 1.044 & -0.122 & 0.041 & 0.071 & -0.200 \\
\hline 1.839 & -0.195 & 0.235 & 0.078 & -0.093 \\
\hline 2.062 & -0.060 & 0.100 & -0.071 & -0.416 \\
\hline 5.033 & -0.254 & -0.065 & -0.007 & -0.417 \\
\hline 10.54 & -0.520 & -0.353 & -0.309 & -0.602 \\
\hline 21.11 & -1.429 & -1.215 & -1.153 & -1.130 \\
\hline 30.04 & -2.212 & -1.974 & -1.985 & -1.790 \\
\hline 34.01 & -2.729 & -2.462 & -2.457 & -2.001 \\
\hline 38.20 & -3.542 & -3.429 & -2.985 & -2.616 \\
\hline 42.71 & -9.479 & -8.093 & -6.771 & -3.375 \\
\hline
\end{tabular}

standard deviation and in Table 10 and Figure 13 for the $\mathrm{C} / \mathrm{N}_{0}$ estimation.

The conclusions drawn from this second sampling jitter effects analysis are similar to the first measurements on the correlation peak's amplitude. From Figure 12, one can see that a sampling clock jitter lower than 5 ps does not significantly affect the horizontal error standard deviation nor the $\mathrm{C} / \mathrm{N}_{0}$ estimation. From 5 ps and above, the horizontal error standard deviation degrades slowly and the $\mathrm{C} / \mathrm{N}_{0}$ decreases rapidly. For instance, even a strong signal at $50 \mathrm{~dB} \cdot \mathrm{Hz}$ sees its $\mathrm{C} / \mathrm{N}_{0}$ reduced by about $4 \mathrm{~dB}$ when subject to a sampling clock jitter of around 30 ps. These results demonstrate that there is a strong correlation between the sampling clock jitter and the accuracy of a GPS receiver.

Moreover, the noise added to the receiver by the sampling clock is significant for certain values of sampling clock jitter, as it can be observed in the $\mathrm{C} / \mathrm{N}_{0}$ estimation. As for the effect on the correlation peak, the results show that the four simulated $\mathrm{C} / \mathrm{N}_{0}$ result in similar behavior, except for the $35 \mathrm{~dB} \cdot \mathrm{Hz}$ case where the solution becomes unstable from a sampling clock jitter of $21.11 \mathrm{ps}$. In fact, the receiver is having difficulty tracking the GPS signal because the $\mathrm{C} / \mathrm{N}_{0}$ is very low. Furthermore, using a sampling clock jitter higher than 34.01 ps on the current DRFS prototype leads to an unusable architecture. The RxGNSS receiver cannot track any signal and this is due to the ADC which produces erratic data outputs as well as a non-periodic Data Ready signal to the FPGA.

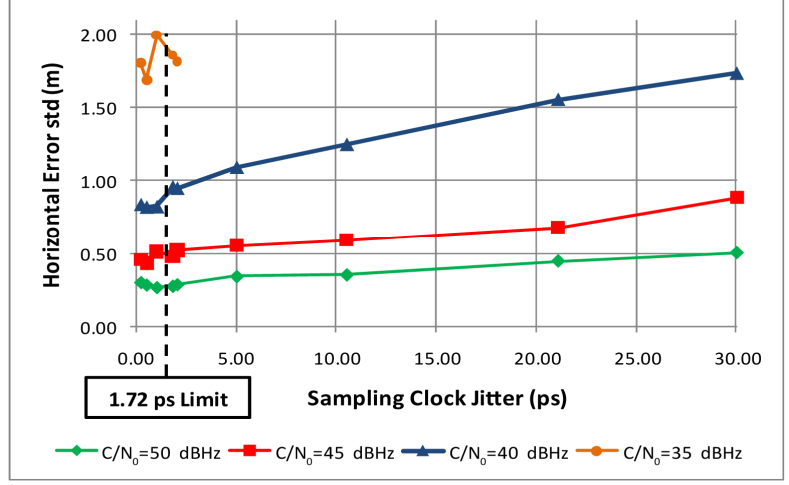

Figure 12. Effects of sampling jitter on the horizontal standard deviation.

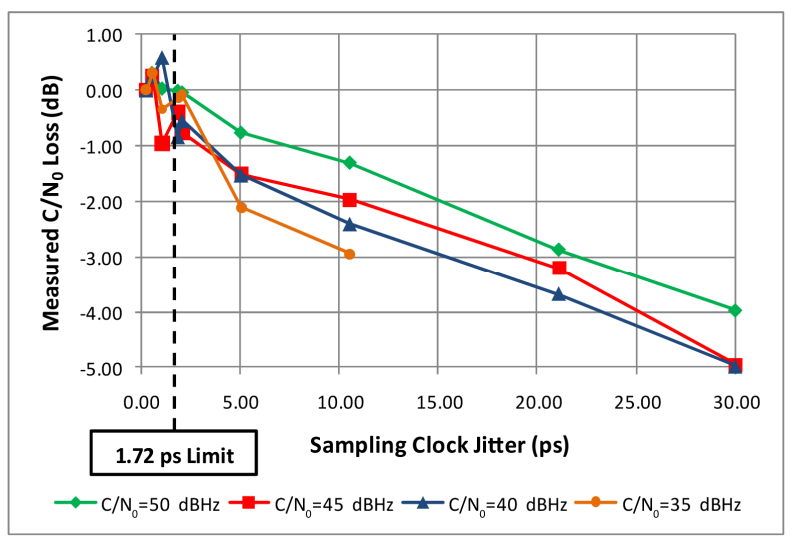

Figure 13. Effects of sampling jitter on the $\mathrm{C} / \mathbf{N}_{0}$. 
Table 9. Effects of sampling jitter on the horizontal error standard deviation-Averaged over 1 hour.

\begin{tabular}{|c|c|c|c|c|c|c|c|c|}
\hline \multirow[b]{3}{*}{ Sampling Clock Jitter (ps) } & \multicolumn{8}{|c|}{$\mathrm{C} / \mathrm{N}_{0}$ of the Simulated GPS Signal } \\
\hline & \multicolumn{2}{|c|}{$50 \mathrm{~dB} \cdot \mathrm{Hz}$} & \multicolumn{2}{|c|}{$45 \mathrm{~dB} \cdot \mathrm{Hz}$} & \multicolumn{2}{|c|}{$40 \mathrm{~dB} \cdot \mathrm{Hz}$} & \multicolumn{2}{|c|}{$35 \mathrm{~dB} \cdot \mathrm{Hz}$} \\
\hline & $\sigma(\mathrm{m})$ & $\Delta(\%)$ & $\sigma(\mathrm{m})$ & $\Delta(\%)$ & $\sigma(\mathrm{m})$ & $\Delta(\%)$ & $\sigma(\mathrm{m})$ & $\Delta(\%)$ \\
\hline 0.243 & 0.301 & $0.00 \%$ & 0.455 & $0.00 \%$ & 0.838 & $0.00 \%$ & 1.8072 & $0.00 \%$ \\
\hline 0.532 & 0.284 & $-1.72 \%$ & 0.432 & $-2.36 \%$ & 0.820 & $-1.80 \%$ & 1.6877 & $-11.95 \%$ \\
\hline 1.044 & 0.267 & $-3.39 \%$ & 0.511 & $5.54 \%$ & 0.824 & $-1.47 \%$ & 1.9942 & $18.70 \%$ \\
\hline 1.839 & 0.276 & $-2.48 \%$ & 0.478 & $2.25 \%$ & 0.959 & $12.07 \%$ & 1.8571 & $4.99 \%$ \\
\hline 2.062 & 0.287 & $-1.42 \%$ & 0.520 & $6.49 \%$ & 0.950 & $11.11 \%$ & 1.8171 & $0.99 \%$ \\
\hline 5.033 & 0.342 & $4.05 \%$ & 0.554 & $9.90 \%$ & 1.093 & $25.42 \%$ & & \\
\hline 10.54 & 0.353 & $5.19 \%$ & 0.591 & $13.57 \%$ & 1.249 & $41.01 \%$ & \multirow{2}{*}{\multicolumn{2}{|c|}{ Unstable Solution }} \\
\hline 21.11 & 0.447 & $14.59 \%$ & 0.679 & $22.40 \%$ & 1.555 & $71.62 \%$ & & \\
\hline 30.04 & 0.503 & $20.20 \%$ & 0.885 & $42.93 \%$ & 1.733 & $89.44 \%$ & & \\
\hline 34.01 & \multicolumn{8}{|c|}{ No Signal } \\
\hline
\end{tabular}

Table 10. Effects of sampling jitter on the $\mathrm{C} / \mathrm{N}_{0}$ measurement-Averaged over 1 hour.

\begin{tabular}{ccccccccc}
\hline & \multicolumn{7}{c}{$\mathrm{C} / \mathrm{N}_{0}$ of the Simulated GPS Signal } \\
\cline { 2 - 8 } & \multicolumn{7}{c}{$50 \mathrm{~dB} \cdot \mathrm{Hz}$} & \multicolumn{2}{c}{$45 \mathrm{~dB} \cdot \mathrm{Hz}$} & \multicolumn{2}{c}{$40 \mathrm{~dB} \cdot \mathrm{Hz}$} & \multicolumn{2}{c}{$35 \mathrm{~dB} \cdot \mathrm{Hz}$} \\
\hline Sampling Clock Jitter $(\mathrm{ps})$ & $\mathrm{C} / \mathrm{N}_{0}(\mathrm{~dB} \cdot \mathrm{Hz})$ & $\Delta(\mathrm{dB})$ & $\mathrm{C} / \mathrm{N}_{0}(\mathrm{~dB} \cdot \mathrm{Hz})$ & $\Delta(\mathrm{dB})$ & $\mathrm{C} / \mathrm{N}_{0}(\mathrm{~dB} \cdot \mathrm{Hz})$ & $\Delta(\mathrm{dB})$ & $\mathrm{C} / \mathrm{N}_{0}(\mathrm{~dB} \cdot \mathrm{Hz})$ & $\Delta(\mathrm{dB})$ \\
\hline 0.243 & 51.07 & 0.00 & 47.52 & 0.00 & 42.69 & 0.00 & 37.99 & 0.00 \\
0.532 & 51.37 & 0.30 & 47.76 & 0.24 & 42.94 & 0.25 & 38.31 & 0.32 \\
1.044 & 51.09 & 0.02 & 46.56 & -0.96 & 43.28 & 0.59 & 37.65 & -0.34 \\
$\mathbf{1 . 8 3 9}$ & $\mathbf{5 1 . 0 5}$ & $-\mathbf{0 . 0 2}$ & $\mathbf{4 7 . 1 2}$ & $-\mathbf{0 . 4 0}$ & $\mathbf{4 1 . 8 5}$ & $-\mathbf{0 . 8 4}$ & $\mathbf{3 7 . 8 5}$ & $-\mathbf{0 . 1 4}$ \\
2.062 & 51.02 & -0.05 & 46.75 & -0.77 & 42.14 & -0.55 & 37.91 & -0.08 \\
5.033 & 50.30 & -0.77 & 46.01 & -1.51 & 41.16 & -1.53 & 35.88 & -2.11 \\
10.54 & 49.76 & -1.31 & 45.55 & -1.97 & 40.28 & -2.41 & 35.04 & -2.95 \\
21.11 & 48.20 & -2.87 & 44.31 & -3.21 & 39.01 & -3.68 & Unstable Solution \\
30.04 & 47.09 & -3.98 & 42.56 & -4.96 & 37.70 & -4.99 & &
\end{tabular}

Position accuracy is the basic and the most widely used performance metric of a GNSS receiver. For highend receivers requiring very high accuracy, the phase of the GNSS carrier is measured and refines the pseudorange position estimation. The jitter effects on the carrier phase is thus of great interest and is the next step forward to validate the jitter limits set by [12]. Studies on this subject are ongoing at the LACIME Laboratory using double difference measurements, the second measurement coming from a NovAtel DL-4 Plus receiver, and will be the subject of a future publication.

\section{Conclusions}

The prototype presented in this paper may be seen as a first step towards the realization of a universal GNSS front-end. The paper shows that such a universal architecture may bring significant valuable benefits for future versions of compact universal GNSS receivers. This GNSS receiver is expected to receive and demodulate the signals from various positioning systems, thus gaining in 
precision, availability and robustness. By using the direct RF sampling technique, the proposed prototype samples a wide frequency range, out of which the GPS L1 C/A signal is processed digitally in this paper. This approach has the advantage of eliminating the conventional superheterodyne frequency down conversion and thus reduces the complexity of the RF front-end when capturing signals in various frequency ranges. By using a high frequency analog-to-digital converter, wideband RF amplifying chain as well as high speed digital signal processing architecture, the prototype demonstrated the real-time feasibility of the DRFS technique for GNSS signals. Indeed, experimental results with a real GPS signal demonstrated that the prototype, with its outputs connected to the RxGNSS, has similar accuracy to that of the worldclass state-of-the-art Novatel DL-4 Plus receiver, which uses the so-called conventional frequency down-conversion technique.

The DRFS is known to be more susceptible to sampling jitter than the conventional superheterodyne architecture and various papers present mathematical models for the limit of the jitter in order not to deteriorate the GNSS signals' $\mathrm{C} / \mathrm{N}_{0}$. [12] sets an upper bound by limiting the jitter noise power to $10 \mathrm{~dB}$ below that of the thermal noise power in the data bandwidth of the GNSS signal being analyzed, corresponding to $1.72 \mathrm{ps}$ for the GPS L1 C/A signal. Using the DRFS prototype, real GPS L1 C/A signals were captured using a clock source having various jitter levels down to $0.243 \mathrm{ps}$, thanks to the prototype's very low intrinsic jitter. The experimentation demonstrated that the signal's $\mathrm{C} / \mathrm{N}_{0}$ as well as the horizontal error are both affected by the sampling jitter. Jitter adds noise to a GNSS receiver and thus decreases the $\mathrm{C} / \mathrm{N}_{0}$, consequently reducing the positioning accuracy and limiting the number of satellites' signals that can be captured. This also results in a higher time to first fix (TTFF). Nevertheless, the sampling jitter limit was proven to be sufficient since no significant degradation was observed for sampling jitters below 1.72 ps. Very high sampling jitter (higher than $30 \mathrm{ps)} \mathrm{resulted} \mathrm{in} \mathrm{an}$ unstable navigation solution, especially for a signal already having a low $\mathrm{C} / \mathrm{N}_{0}$ of around $35 \mathrm{~dB} \cdot \mathrm{Hz}$.

The DRFS prototype presented in this paper is currently under further developments in order to extend its capabilities by capturing more GNSS signals located in different frequency bands. In the end, the goal is to sample the entire GNSS band (the combined G1 and G2-5 bands) and to use as many positioning signals as possible. Future work also includes the experimentation of the jitter effects on the carrier phase measurements using a double difference technique.

\section{Acknowledgements}

This work was supported financially with master school- arships from the Natural Sciences and Engineering Research Council of Canada (NSERC), the Fonds Québécois de la Recherche sur la Nature et les Technologies (FQRNT), the École de Technologie Supérieure (ÉTS) and by the Laboratoire de Communication et D'intégration de la Microélectronique (LACIME).

\section{REFERENCES}

[1] S. Clark, "Soyuz Launch Restores Russian Navigation System," 2011.

http://www.spaceflightnow.com/news/n1110/02soyuz/

[2] B. Peter de Selding, "Soyuz Lofts Two Galileo Satellites in Debut from European Spaceport," 2011.

http://www.spacenews.com/launch/111021-soyuz-lofts-g alileo-sats-debut.html

[3] S. Clark, "Beidou Navigation Payload Launched by Chinese Rocket,” 2012.

http://spaceflightnow.com/news/n1202/24longmarch/

[4] B. Sauriol, "Mise en Oeuvre en Temp-Réel d'un Récepteur Hybride GPS-Galileo," Master's Thesis, École de Technologie Supérieure, Montréal, 2008.

[5] A. Brown and W. Sward, "Digital Down Conversion Test Results with a Broadband L-band GPS Receiver," AIAA/ IEEE Digital Avionics Systems Conference, 13th DASC, Colorado Springs, 30 October-3 November 1994, pp. 426-431. doi:10.1109/DASC.1994.369446

[6] J. Thor and D. M. Akos, "A Direct RF Sampling Multifrequency GPS Receiver," IEEE Position Location and Navigation Symposium, Palm Springs, 15-18 April 2002, pp. 44-51. doi:10.1109/PLANS.2002.998887

[7] M. L. Psiaki, D. M. Akos and J. T. Thor, "A Comparison of 'Direct RF Sampling' and 'Down-Convert and Sampling' GNSS Receiver Architectures," Journal of the Institute of Navigation, Vol. 52, No. 2, 2005, pp. 71-81.

[8] D. M. Akos, M. Stockmaster, J. B. Y. Tsui and J. Caschera, "Direct Bandpass Sampling of Multiple Distinct RF Signals," IEEE Transactions on Communications, Vol. 47, No. 7, 1999, pp. 983-988. doi:10.1109/26.774848

[9] M. L. Psiaki, S. P. Powell, H. Jung and P. M. Kintner, "Design and Practical Implementation of Multifrequency RF Front Ends Using Direct RF Sampling," IEEE Transactions on Microwave Theory and Techniques, Vol. 53, No. 10, 2005, pp. 3082-3089. doi:10.1109/TMTT.2005.855127

[10] A. Dempster, “Aperture Jitter Effects in Software Radio GNSS Receivers," Journal of Global Positioning Systems, Vol. 3, No. 1-2, 2004, pp. 45-48. doi:10.5081/jgps.3.1.45

[11] A. Dempster and A. Bilal, "Aperture Jitter Effects in Software Radio GNSS Receivers," International Global Navigation Satellite Systems Society Symposium, Australia, 17-21 July 2006, pp. 1-15.

[12] A. Dempster, “Aperture Jitter in BPSK Systems," IEEE Electronics Letters, Vol. 41, No. 6, 2005, pp. 371-373. doi:10.1049/el:20057416

[13] M. Weeks, "Digital Signal Processing Using Matlab and Wavelets," 1st Edition, Infinity Science Press, Hingham, 
2007.

[14] J. Bae and J. Park, "An Efficient Algorithm for Bandpass Sampling of Multiple RF Signals," IEEE Signal Processing Letters, Vol. 13, No. 4, 2006, pp. 193-196. doi:10.1109/LSP.2005.863694

[15] S. Sen and V. M. Gadre, "An Algorithm for Minimum Bandpass Sampling Frequency for Multiple RF Signal in SDR System," IEEE/SP 13th Workshop on Statistical Signal Processing, Bordeaux, 17-20 July 2005, pp. 327332. doi:10.1109/SSP.2005.1628615

[16] C.-H. Tseng and S.-C. Chou, "Direct Downconversion of Multiple RF Signals Using Bandpass Sampling," IEEE International Conference on Communications, Anchorage, 11-15 May 2003, pp. 2003-2007. doi:10.1109/ICC.2003.1203950

[17] M. Choe and K. Kim, "Bandpass Sampling Algorithm with Normal and Inverse Placements for Multiple RF Signals," IEICE Transactions on Communications, Vol. E88-B, No. 2, 2005, pp. 754-757. doi:10.1093/ietcom/E88-B.2.754

[18] A. G. Dempster, "Quadrature Bandpass Sampling Rules for Single and Multiband Communications and Satellite Navigation Receivers," IEEE Transaction on Aerospace and Electronic Systems, Vol. 47, No. 4, 2011, pp. 23082316. doi:10.1109/TAES.2011.6034634

[19] C. Barret, "Fractionnal/Integer-N PLL Basics," Texas In- struments Inc., Dallas, 1999.

[20] B. Le, T. W. Rondeau, J. H. Reed and C. W. Bostian, “Analog-to-Digital Converters," IEEE Signal Processing Magazine, Vol. 22, No. 6, 2005, pp. 69-77. doi:10.1109/MSP.2005.1550190

[21] G. Lamontagne, "Conception et Mise en Oeuvre d'Une Tête de Réception à Échantillonnage Direct RF pour les Signaux de Radionavigation par Satellites," Master Thesis in Electrical Engineering, École de Technologies Supérieure, Montréal, 2009.

[22] E. D. Kaplan and C. Hegarty, "Understanding GPS Principles and Applications," 2nd Edition, Artech House, Boston, 2006.

[23] M.-A. Fortin, J.-C. Guay and R. J. Landry, "Development of a Universal GNSS Tracking Channel," Proceedings of the 22nd International Technical Meeting of the Satellite Division of the Institute of Navigation ION GNSS 2009, Savannah, 22-25 September 2009, pp. 259-272.

[24] R. Landry Jr., M.-A. Fortin and J.-C. Guay, "Universal Acquistion and Tracking Apparatus for Global Navigation Satellite System (GNSS)," USPTO, Alexandria, 2010.

[25] T. Bose, "Digital Signal and Image Processing," 1st Edition, John Wiley and Sons Ltd., Hoboken, 2004.

[26] K. Yang, "Phase-Noise Profiles Aid System Testing," $R F$ Design, 2005. http://rfdesign.com/mag/511RFDF2.pdf 